\title{
Improving COPD Care at the University of North Carolina Internal Medicine Outpatient Clinic
}

\author{
Camilla S. Powierza, MD', Nelson Vick, BS', Brenna K. McManus, BA', \\ and Amy W. Shaheen, MD, MSc'
}

\author{
Mary Reich Cooper, MD, JD, Abdurahman Alloghbi, MD, CMQ, \\ Jason D. Hall, MD, JD, CMQ, Michael X. Jin, BS, and \\ Lukman Lawal, MD, MPH, Section Editors
}

The Global Initiative for Chronic Obstructive Lung Disease has encouraged symptom assessment for all chronic obstructive pulmonary disease (COPD) patients and pulmonary rehabilitation for COPD patients with high symptom burden and risk of exacerbations. ${ }^{1}$ Pulmonary rehabilitation has been shown to improve their symptoms, quality of life, and exercise tolerance and to reduce readmissions and mortality in patients with a recent exacerbation ( $\leq 4$ weeks from prior hospitalization). ${ }^{2,3}$ However, many patients never have the opportunity to take advantage of pulmonary rehabilitation because systematic ways of assessing symptoms, identifying symptomatic patients, and referring those who would benefit from pulmonary rehabilitation are lacking. In the University of North Carolina general medicine clinic, symptom assessment and pulmonary rehabilitation referrals were not done prior to this effort. The project's primary goal was to systematically screen for dyspnea symptoms during all COPD patient encounters, with secondary goals of improving rates of screening for hypoxia, referrals to pulmonary rehabilitation in symptomatic patients, and documentation in the electronic health record.

To address these goals, a quality improvement team was formed including a medical student, patient educator, front desk staff, and a physician. The process began with a staff member flagging charts for patients due for symptom assessment. The front desk responded to the flag and distributed a paper copy of the Modified Medical Research Council (mMRC) dyspnea scale to patients at check-in. ${ }^{4}$ Patients self-scored their degree of dyspnea (grades 0-4). After completing the form, patient scores $\geq 2$ prompted algorithmic responses by members of the health care team. Nurses checked oxygen saturations for symptomatic patients. Provider algorithms included escalating inhaler therapy and placing pulmonary rehabilitation referrals. The process was further streamlined with daily messages to providers who had COPD patients scheduled and provider education sessions on pulmonary rehabilitation and the cost-effective benefits for patients.

Over the span of 42 weeks, there were 571 COPD patient encounters, $87 \%$ of which were screened for symptoms of dyspnea with an mMRC questionnaire. Of these patients, 233 were found to be highly symptomatic $(\mathrm{mMRC} \geq 2)$ and qualified for pulmonary rehabilitation, with a total of 30 referrals to pulmonary rehabilitation made $(30 / 233=13 \%)$. Oxygen saturation was checked in $78 \%$ of these symptomatic patients. MMRC scoring was documented with a note template available to all providers, and during the 42 weeks, scores were documented 126 times $(126 / 571=22 \%)$, from a baseline of $0 \%$.

Overall, COPD symptom assessment has improved by using an mMRC questionnaire; an algorithm of care was followed with good fidelity by providers and nurses. The rate of pulmonary rehabilitation referrals increased over 42 weeks and there was a substantial increase in awareness of pulmonary rehabilitation as a treatment option for symptomatic COPD patients. Ease of documentation has continued to improve with automatic reminders in the electronic health record. Future directions involve improving rates of referral to pulmonary rehabilitation and understanding its limitations.

\section{References}

1. Global Initiative for Chronic Obstructive Lung Disease. Global strategy for the diagnosis, management and prevention of chronic obstructive pulmonary disease; 2018 report. https://goldcopd.org/wp-content/uploads/2017/11/GOLD -2018-v6.0-FINAL-revised-20-Nov_WMS.pdf. Published 2018. Accessed July 30, 2018.

2. McCarthy B, Casey D, Devane D, Murphy K, Murphy E, Lacasse Y. Pulmonary rehabilitation for chronic obstructive pulmonary disease. Cochrane Database Syst Rev. 2015;(2): CD003793.

3. Puhan MA, Gimeno-Santos E, Scharplatz M, Troosters T, Walters $\mathrm{EH}$, -Steurer J. Pulmonary rehabilitation following exacerbations of chronic obstructive pulmonary disease. Cochrane Database Syst Rev. 2011;(10):CD005305.

4. Mahler DA, Wells CK. Evaluation of clinical methods for rating dyspnea. Chest. 1988;93:580-586.

'University of North Carolina, Chapel Hill, NC

Corresponding Author:

Camilla S. Powierza, MD, Department of Internal Medicine, University of North Carolina School of Medicine, CB\#7I 10, 101 Manning Dr, Chapel Hill, NC 27599.

Email: camilla.powierza@yale.edu 